\title{
Aplicación de las TIC en los profesores del área de las ciencias sociales: derecho y economía
}

\author{
APPLICATION OF ICT IN TEACHERS IN THE AREA OF SOCIAL SCIENCES: LAW
} AND ECONOMICS

\author{
Daniel Claudio Perazzo Logioia ${ }^{1}$, María Teresa Pantoja Sánchez² \\ 1. Vicerrector del Instituto Superior Tecnológico Particular Sudamericano, Cuenca. \\ Email: vicerectoradoits@sudamericano.edu.ec ORCID: https://orcid.org/0000-0003-3089-2225
}

2. Comité Permanente de Autoevaluación Institucional del Instituto Superior Tecnológico Particular Sudamericano-Profesora de la Universidad Nacional de Educación, Cuenca. Email: mtpantoja@sudamericano.edu.ec ORCID: https://orcid.org/0000-0002-3241-6241

Para Citar: Perazzo Logioia, D. C., \& Pantoja Sánchez, M. T. (2020). Aplicación de las TIC en los profesores del área de las ciencias sociales: derecho y economía. Revista Publicando, 7(25), 180-192. Recuperado a partir de https://revistapublicando.org/revista/index.php/crv/article/view/2117

\begin{abstract}
Resumen: Este estudio de tipo descriptivo examina la aplicación de las TIC en los profesores pertenecientes a carreras del ámbito de las ciencias sociales en una universidad pública mexicana. Las áreas académicas que intervienen son las de Derecho y Economía. El artículo tiene como referente una investigación más amplia que se hizo extensiva a facultades como Medicina, Ingeniería y Agronomía. La aplicación de un cuestionario a 38 profesores que imparten asignaturas en estas carreras nos ha llevado a determinar en qué grado se utilizan las tecnologías en clase, los objetivos que consideran a la hora de implementarlas y las actitudes que presentan los profesores en relación a las TIC. Como afirma (Prats, 2016) "La cuestión no es estar a favor o en contra de la introducción de las tecnologías en la educación, ya que todos estamos a favor. Lo relevante es determinar qué papel deben jugar en un proceso de enseñanza aprendizaje". Para Salvador (1991) la computadora es un medio innovador, que provoca "...conseguir el aprendizaje como un material más dentro del aula" y que no sustituye al profesor, más bien viene a apoyar la labor del docente. Los profesores de una carrera tradicional como Derecho son más reacios a utilizar las TIC en sus clases al igual que aquellos que forman parte del programa académico de Economía. Sin embargo, a casi 20 años del nuevo siglo es inexcusable que el docente reciba cursos y talleres que apoyen su labor cotidiana, con objeto de poder conocer y aplicar las TIC en consonancia con las exigencias del mundo laboral en el cual se desempeñará el alumno en un futuro próximo.
\end{abstract}

Palabras clave: TIC, actitudes pedagógicas, innovación tecnológica, formación docente.
Abstract: This descriptive study examines the application of ICT in teachers' careers from the field of social sciences in a Mexican public university. Academic areas involved are law and economics. This article is concerning a broader research that extended to faculties such as Medicine, Engineering and Agronomy. The application of a questionnaire to 38 teachers who teach subjects in these careers, has led us to determine the extent to which technologies are used in class, the objectives considered when implementing, and attitudes presented by teachers in relation to TIC. (Prats, 2016) states, "The question is not to be for or against the introduction of technology in education, and we are all in favor. What is relevant is to determine what role should they play in a teaching-learning process. "According to Salvador (1991) a computer is an innovative means which causes "....achieve learning as another material in the classroom" and that does not replace the teacher, rather come to support the work of teachers. Teachers' career as a traditional law, are more reluctant to use ICT in their classes as well as those who are part of the academic program of Economy. However, nearly 20 years of the new century is inexcusable that teachers receive courses and workshops to support their daily work in order to be able to understand and apply ICT in line with the demands of work in which the student will perform in the near future.

Keywords: ICT, pedagogical attitudes, technological innovation, teacher training. 


\section{INTRODUCCIÓN}

$\mathrm{E}$ 1 trabajo que presentamos tiene como referente una amplia investigación que indaga sobre los usos y actitudes de los profesores que pertenecen a diferentes facultades de una universidad pública mexicana. Este artículo está centrado en una de las facultades en donde se llevó a cabo la revisión de los datos, la Facultad de Ciencias Sociales, en donde se recabó información de los profesores que imparten clases en carreras como Derecho y Economía. Pretendemos explicar y argumentar a través de la aplicación de una encuesta cómo los docentes propician el uso de la tecnología en clases, así como los objetivos que consideran para su aplicación y las actitudes que despiertan en ellos la utilización de las TIC.

Los objetivos de este estudio son:

- Identificar los recursos tecnológicos que utiliza el profesor en sus clases.

- Apreciar la importancia que le brinda el docente a las TIC para fortalecer el proceso de enseñanza-aprendizaje de sus alumnos.

- Definir la categoría de objetivos que el docente considera para utilizar las TIC en la asignatura que imparte.

- Valorar las actitudes de los profesores en relación con las TIC.

Para que un profesor pueda encauzar a sus alumnos, primero debe estar preparado para ello, como dice Perazzo (1995)

En cierta medida, todos sabemos lo necesario de esta herramienta, en estos últimos años, en que toda la información se encuentra computarizada. Ejemplo de ello son los programas informáticos que nos presentan un gran paquete informativo: enciclopedias, por ejemplo, en entorno PC y bajo la modalidad de los sistemas multimedia... Las reformas educativas de los últimos años promueven, la implementación de las nuevas tecnologías. Es público que las reformas en un sistema educativo tienen como objetivo prioritario mejorar la calidad de la enseñanza y adecuarla a las nuevas demandas sociales.

Bajo esta perspectiva consideramos que se hace obligatorio brindarles a los docentes un conocimiento que guíe sus decisiones curriculares y ofrezca sugerencias para la práctica educativa. Es urgente fortalecer la formación técnica pedagógica, la cual consistiría en una aproximación amplia a las posibilidades educativas que ofrecen las TIC, contextualizadas en distintos modelos de enseñanza y aprendizaje. No dejemos de lado la formación instrumental, es decir, la familiarización con la computadora y todos los periféricos necesarios con los programas más adecuados para utilizar todo el software disponible de la forma más conveniente, sin que atente con la identidad profesional del profesor, ni le cree inseguridad. Es preciso promover una formación docente autorreflexiva a partir del análisis del rol del profesor, en un contexto en el cual los alumnos poseen amplios conocimientos de tecnología, muchas veces sin aplicarla al medio educativo y que pudieran favorecer su aprendizaje. Esto viene dado por la falta de experiencia y conocimiento en el docente para hacer uso adecuado de ella en la asignatura que imparte.

\section{Utilización de las TIC en el Aula}

Cada vez vemos mayor número de alumnos que saben acceder de forma rápida a grandes cantidades de información, ya que se encuentran acostumbrados a un lenguaje global. El desarrollo tecnológico ha provocado el auge de nuevas habilidades en los estudiantes que deben ser tenidos en cuenta. Por lo tanto, los profesores deben asumir la utilización de las nuevas tecnologías en el trabajo sin dejar de mencionar que deberán estar apoyados por las autoridades educativas, tanto en su propia preparación profesional, como en la dotación de tecnologías educativas en el ámbito institucional.

La gran cantidad de información que se mueve en nuestro entorno ha dejado en manos de educadores una larga tradición centrada en la transmisión de información como herramienta de enseñanza-aprendizaje. En la actualidad, hay un mayor número de información que aparece en internet y en las redes, con lo cual los jóvenes de hoy tienen un acceso ilimitado a ella. El uso de los medios informáticos en el proceso de enseñanza-aprendizaje no es nuevo, pero la frecuencia y tiempo con que los alumnos los usan están relacionados directamente con su edad. Actualmente la educación en los diferentes centros de estudios, sean de carácter infantil, de enseñanza media o superior, se encuentra 
encaminada hacia la competencia de "aprender a aprender", e intenta dejar a un lado la acumulación de información para acercarse al saber utilizar herramientas y recursos que permitan a los alumnos a aprender, concretar qué información se necesita en cada momento, cómo localizarla, recuperarla, procesarla, reelaborarla, cómo comunicar sus percepciones en torno a ella, extraer el conocimiento necesario en cada momento y para cada situación y construir el propio conocimiento sobre la base de una búsqueda activa. Por lo tanto, como nos indican Hernández et al., (2014): “El uso efectivo de las TIC en el aula depende de la efectividad y eficacia de los procesos de enseñanza-aprendizaje desarrollados y de la capacidad de los docentes y estudiantes para interactuar con los recursos tecnológicos".

\section{METODOLOGÍA}

"La ciencia no tiene el monopolio de la verdad, sino sólo el de los medios para someter la verdad a prueba y reforzarla"

Mario Bunge

Consideramos que la metodología más acorde al tipo de estudio que planteamos es la cuantitativa, con objeto de obtener información de primera mano de los profesores encuestados. Se llevó a cabo un análisis cruzado,

...lo cual implica una representación tabular de datos numéricos, por lo general representados en forma de frecuencia y porcentaje, en la cual las variables se dividen en forma cruzada para estudiar sus relaciones. Este análisis cruzado permite al investigador determinar la naturaleza de las relaciones entre las variables (Ortiz, 2016, p.15).

Para ello aplicamos un instrumento con 25 reactivos a 38 profesores de la Facultad de Ciencias Sociales de una universidad pública mexicana, los cuales forman parte de las carreras de Derecho (24) y Economía (14).

\section{RESULTADOS}

\section{1. Área Académica: Derecho}

Se observa el porcentaje de hombres y mujeres encuestados, siendo el total de 24 .
Gráfica 1. Sexo.

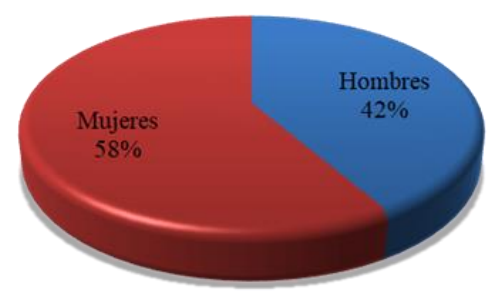

Fuente: Perazzo y Pantoja (2020).

El rango de edades que más sobresale es el comprendido entre los 30-40 años, lo que indica ser una población de profesores jóvenes.

Tabla 1. Edad.

\begin{tabular}{lll}
\hline Rango/años & Frecuencia & Porcentaje \\
\hline $22-26$ & 7 & 29,2 \\
$27-29$ & 2 & 8,3 \\
$30-34$ & 9 & 37,5 \\
$35-40$ & 2 & 8,3 \\
$>40$ & 4 & 16,7 \\
Total & $\mathbf{2 4}$ & $\mathbf{1 0 0 , 0}$ \\
\hline
\end{tabular}

Fuente: Perazzo y Pantoja (2020).

En cuanto al nivel de estudios alcanzados podemos observar que ninguno posee estudios de Doctorado, prevaleciendo los estudios pertenecientes al tercer nivel.

Gráfica 2. Nivel de Estudios Alcanzado.

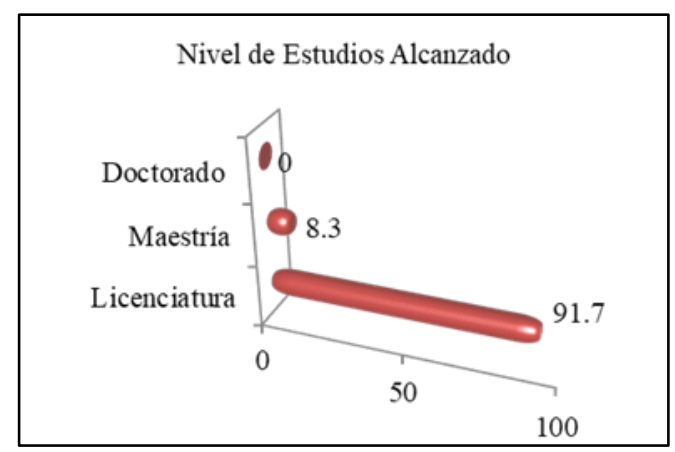

Fuente: Perazzo y Pantoja (2020). 
El tiempo de servicios que viene prestando en el área es de 1 a 5 años en su mayoría. El $45.8 \%$ son profesores de medio tiempo, mientras que el $37.5 \%$ poseen nombramiento como profesores de tiempo completo. Con respecto a las tecnologías que se usan en clase, como podemos observar en la tabla 2, sólo las transparencias, diapositivas, filmes/video y televisión tienen una moda de 3 lo cual implica que algunas veces los usan, mientras todas las demás herramientas muestran una moda de 1 y ello indica que nunca o pocas veces las utilizan.

Tabla 2. Uso de las tecnologías en clases.

\begin{tabular}{|c|c|c|c|c|c|c|c|c|c|c|c|c|c|c|}
\hline \multirow[t]{2}{*}{ Categoría } & \multicolumn{6}{|c|}{ Porcentaje } & \multicolumn{6}{|c|}{ Frecuencia } & \multirow[t]{2}{*}{ Moda } & \multirow[t]{2}{*}{ Media } \\
\hline & 0 & 1 & 2 & 3 & 4 & 5 & 0 & 1 & 2 & 3 & 4 & 5 & & \\
\hline Transparencias & 0,0 & 16,7 & 29,2 & 45,8 & 8,3 & 0,0 & 0 & 4 & 7 & 11 & 2 & 24 & 3 & 2,46 \\
\hline Diapositivas & 0,0 & 33,3 & 20,8 & 37,5 & 8,3 & 0,0 & 0 & 8 & 5 & 9 & 2 & 0 & 3 & 2,21 \\
\hline Videogramas & 0,0 & 37,5 & 16,7 & 33,3 & 8,3 & 4,2 & 0 & 9 & 4 & 8 & 2 & 1 & 1 & 2,25 \\
\hline Filmes/Video & 0,0 & 20,8 & 33,3 & 29,2 & 8,3 & 8,3 & 0 & 5 & 8 & 7 & 2 & 2 & 2 & 2,50 \\
\hline Televisión & 0,0 & 25,0 & 20,8 & 33,3 & 16,7 & 4,2 & 0 & 6 & 5 & 8 & 4 & 1 & 3 & 2,54 \\
\hline Videodiscos & 4,2 & 45,8 & 12,5 & 25,0 & 8,3 & 4,2 & 1 & 11 & 3 & 6 & 2 & 1 & 1 & 2,00 \\
\hline Grabaciones sonoras & 0,0 & 50,0 & 8,3 & 25,0 & 12,5 & 4,2 & 0 & 12 & 2 & 6 & 3 & 1 & 1 & 2,13 \\
\hline Computadoras & 0,0 & 29,2 & 33,3 & 20,8 & 12,5 & 4,2 & 0 & 7 & 8 & 5 & 3 & 1 & 2 & 2,29 \\
\hline Materiales & 0,0 & 45,8 & 29,2 & 16,7 & 8,3 & 0,0 & 0 & 11 & 7 & 4 & 2 & 0 & 1 & 1,88 \\
\hline \multicolumn{15}{|c|}{ Hipermedia/Multimedia } \\
\hline Radio & 0,0 & 62,5 & 25,0 & 0,0 & 12,5 & 0,0 & 0 & 15 & 6 & 0 & 3 & 0 & 1 & 1,63 \\
\hline Datashow & 0 & 66,7 & 16,7 & 8,3 & 8,3 & 0,0 & 0 & 16 & 4 & 2 & 2 & 0 & 1 & 1,58 \\
\hline Software tutorial & 0,0 & 70.8 & 8,3 & 16,7 & 4,2 & 0,0 & 0 & 17 & 2 & 4 & 1 & 0 & 1 & 1,54 \\
\hline Power Point & 0,0 & 33,3 & 25,0 & 25,0 & 12,5 & 4,2 & 0 & 8 & 6 & 6 & 3 & 1 & 1 & 2,29 \\
\hline Software general & 0,0 & 29,2 & 12,5 & 25,0 & 29,2 & 4,2 & 0 & 7 & 3 & 6 & 7 & 1 & 2 & 2,67 \\
\hline Photoshop & 4,2 & 70.8 & 8,3 & 12,5 & 4,2 & 0,0 & 1 & 17 & 2 & 3 & 1 & 0 & 1 & 1,42 \\
\hline
\end{tabular}

Fuente: Perazzo y Pantoja (2020).

La mayoría de los objetivos como puede observarse en la tabla 3, recibieron puntuaciones altas con una moda de entre 4 y 5 , lo cual revela que muchas veces y siempre se usan las TIC para alcanzar estos objetivos. Sin embargo, memorizar y gestión de recursos recibieron la puntuación más baja, lo que indica que nunca se persiguen estos objetivos.

Por último, los objetivos relacionar/vincular/ordenar, concientizar, sintetizar, propiciar nuevas relaciones y retroalimentar, sólo se consideran algunas veces. 
Tabla 3. Objetivos en el Uso de las TIC.

\begin{tabular}{|c|c|c|c|c|c|c|c|c|c|c|c|c|c|c|}
\hline \multirow[t]{2}{*}{ Categoría } & \multicolumn{6}{|c|}{ Porcentaje } & \multicolumn{6}{|c|}{ Frecuencia } & \multirow[t]{2}{*}{ Moda } & \multirow[t]{2}{*}{ Media } \\
\hline & 0 & 1 & 2 & 3 & 4 & 5 & 0 & 1 & 2 & 3 & 4 & 5 & & \\
\hline Motivar e interesar & 0,0 & 0,0 & 4,2 & 33,3 & 37,5 & 25,0 & 0 & 0 & 1 & 8 & 9 & 6 & 4 & 3,83 \\
\hline Percibir lo complejo y lo abstracto & 0,0 & 20,8 & 4,2 & 25,0 & 33,3 & 16,7 & 0 & 5 & 1 & 6 & 8 & 4 & 4 & 3,21 \\
\hline Documentar & 0,0 & 0,0 & 8,3 & 33,3 & 33,3 & 25,0 & 0 & 0 & 2 & 8 & 8 & 6 & 4 & 3,75 \\
\hline Memorizar & 0,0 & 54,2 & 16,7 & 12,5 & 12,5 & 4,2 & 0 & 13 & 4 & 3 & 3 & 1 & 1 & 1,96 \\
\hline Nuevos aspectos & 0,0 & 0,0 & 4,2 & 41,7 & 29,2 & 25,0 & 0 & 0 & 1 & 10 & 7 & 6 & 3 & 3,75 \\
\hline Relacionar/Vincular/Ordenar & 0,0 & 4,2 & 4,2 & 37,5 & 20,8 & 33,3 & 0 & 1 & 1 & 9 & 5 & 8 & 3 & 3,75 \\
\hline Clases atractivas & 0,0 & 0,0 & 4,2 & 16,7 & 41,7 & 37,5 & 0 & 0 & 1 & 4 & 10 & 9 & 4 & 4,13 \\
\hline Transmitir perspectivas distintas & 0,0 & 0,0 & 8,3 & 33,3 & 25,0 & 33,3 & 0 & 0 & 2 & 8 & 6 & 8 & 4 & 3,83 \\
\hline Concientizar & 0,0 & 4,2 & 0,0 & 37,5 & 33,3 & 25,0 & 0 & 1 & 0 & 9 & 8 & 6 & 3 & 3,75 \\
\hline Investigar & 0,0 & 0,0 & 8,3 & 29,2 & 29,2 & 33,3 & 0 & 0 & 2 & 7 & 7 & 8 & 5 & 3,88 \\
\hline Sintetizar & 0,0 & 4,2 & 16,7 & 54,2 & 4,2 & 20,8 & 0 & 1 & 4 & 13 & 1 & 5 & 3 & 3,21 \\
\hline Retroalimentar & 0,0 & 0,0 & 4,2 & 45,8 & 33,3 & 16,7 & 0 & 0 & 1 & 11 & 8 & 4 & 3 & 3,63 \\
\hline Generar creatividad & 0,0 & 0,0 & 4,2 & 29,2 & 33,3 & 33,3 & 0 & 0 & 1 & 7 & 8 & 8 & 5 & 3,96 \\
\hline Trabajo colaborativo & 0,0 & 4,2 & 20,8 & 20,8 & 37,5 & 16,7 & 0 & 1 & 5 & 5 & 9 & 4 & 4 & 3,42 \\
\hline Propiciar nuevas relaciones & 0,0 & 8,3 & 8,3 & 41,7 & 25,0 & 16,7 & 0 & 2 & 2 & 10 & 6 & 4 & 3 & $3, .33$ \\
\hline Formación y perfeccionamiento & 4,2 & 8,3 & 8,3 & 29,2 & 33,3 & 16,7 & $1 \mid$ & 2 & 2 & 7 & 8 & 4 & 4 & 3,29 \\
\hline $\begin{array}{l}\text { Hacer conocer diversas culturas } \\
\text { existentes }\end{array}$ & 0,0 & 4,2 & 12,5 & 29,2 & 20,8 & 33,3 & 0 & 1 & 3 & 7 & 5 & 8 & 5 & 3,67 \\
\hline Propiciar uso de internet & 0,0 & 4,2 & 20,8 & 20,8 & 29,2 & 25,0 & 0 & 1 & 5 & 5 & 7 & 6 & 4 & 3,50 \\
\hline Gestión de recursos & 4,2 & 29,2 & 12,5 & 25,0 & 25,0 & 4,2 & 1 & 7 & 3 & 6 & 6 & 1 & 1 & 2,50 \\
\hline Organización de clases & 0,0 & 4,2 & 25,0 & 29,2 & 16,7 & 25,0 & 0 & 1 & 6 & 7 & 4 & 6 & 3 & 3,33 \\
\hline Evaluar & 0,0 & 20,8 & 16,7 & 20,8 & 37,5 & 4,2 & 0 & 5 & 4 & 5 & 9 & 1 & 4 & 2,88 \\
\hline
\end{tabular}

Fuente: Perazzo y Pantoja (2020).

La valoración de las actitudes pedagógicas en relación con las TIC muestra que los profesores están siempre de acuerdo con que el aprendizaje es el resultado de un conjunto muy amplio de procesos dinámicos. Así también, expresan estar muy de acuerdo con que la enseñanza es causa directa del aprendizaje, el cual se mide con relación a reglas/patrones, el aprendizaje se evalúa a partir de la aplicación de conocimientos, enseñar bien es lo bastante para aprender bien y contenidos más cercanos para el aprendizaje más eficaz. Con todas las demás actitudes que se enuncian en la tabla 4, los profesores están más o menos de acuerdo. Excepto con la que señala que el profesor prepara pormenorizadamente las respuestas, con la cual están pocas veces de acuerdo. 
Tabla 4. Actitudes de los profesores en relación con las TIC.

\begin{tabular}{|c|c|c|c|c|c|c|c|c|c|c|c|c|c|c|}
\hline \multirow{3}{*}{$\begin{array}{l}\text { Categoría } \\
\text { La enseñanza es causa } \\
\text { directa del aprendizaje }\end{array}$} & \multicolumn{6}{|c|}{ Porcentaje } & \multicolumn{6}{|c|}{ Frecuencia } & \multirow[t]{2}{*}{ Moda } & \multirow[t]{2}{*}{ Media } \\
\hline & 0 & 1 & 2 & 3 & 4 & 5 & 0 & 1 & 2 & 3 & 4 & 5 & & \\
\hline & 4,2 & 8,3 & 16,7 & 33,3 & 33,3 & 4,2 & 1 & 2 & 4 & 8 & 8 & 1 & 4 & 2,96 \\
\hline $\begin{array}{l}\text { El aprendizaje se mide } \\
\text { con relación } \\
\text { reglas/patrones }\end{array}$ & 0,0 & 4,2 & 4,2 & 33,3 & 33,3 & 25,0 & 0 & 1 & 1 & 8 & 8 & 6 & 4 & 3,71 \\
\hline $\begin{array}{l}\text { El aprendizaje es } \\
\text { resultado de un conjunto } \\
\text { de procesos }\end{array}$ & 0,0 & 4,2 & 16,7 & 12,5 & 33,3 & 33,3 & 0 & 1 & 4 & 3 & 8 & 8 & 5 & 3,75 \\
\hline $\begin{array}{l}\text { El aprendizaje se evalúa a } \\
\text { partir de la aplicación de } \\
\text { conocimientos }\end{array}$ & 0,0 & 0,0 & 12,5 & 25,0 & 54,2 & 8,3 & 0 & 0 & 3 & 6 & 13 & 2 & 4 & 3,58 \\
\hline $\begin{array}{l}\text { Enseñar bien es lo } \\
\text { bastante para aprender } \\
\text { bien }\end{array}$ & 0,0 & 4,2 & 4,2 & 33,3 & 33,3 & 25,0 & 0 & 1 & 1 & 8 & 8 & 6 & 4 & 3,71 \\
\hline $\begin{array}{l}\text { Usar la técnica más } \\
\text { apropiada no es } \\
\text { sinónimo de eficacia }\end{array}$ & 4,2 & 4,2 & 25,0 & 37,5 & 16,7 & 12,5 & 1 & 1 & 6 & 9 & 4 & 3 & 3 & 2,96 \\
\hline $\begin{array}{l}\text { El profesor prepara } \\
\text { pormenorizadamente las } \\
\text { respuestas }\end{array}$ & 0,0 & 0,0 & 37,5 & 20,8 & 25,0 & 16,7 & 0 & 0 & 9 & 5 & 6 & 4 & 2 & 3,21 \\
\hline $\begin{array}{l}\text { Contenidos más cercanos } \\
\text { aprendizaje más eficaz }\end{array}$ & 0,0 & 8,3 & 25,0 & 25,0 & 33,3 & 8,3 & 0 & 2 & 6 & 6 & 8 & 2 & 4 & 3,08 \\
\hline $\begin{array}{l}\text { Técnicas bien aplicadas } \\
\text { garantizan la eficacia }\end{array}$ & 0,0 & 4,2 & 20,8 & 33,3 & 20,8 & 20,8 & 0 & 1 & 5 & 8 & 5 & 5 & 3 & 3,33 \\
\hline $\begin{array}{l}\text { El profesor prepara los } \\
\text { recursos } \\
\text { necesarios }\end{array}$ & 0,0 & 8,3 & 20,8 & 37,5 & 25,0 & 8,3 & 0 & 2 & 5 & 9 & 6 & 2 & 3 & 3,04 \\
\hline
\end{tabular}

Fuente: Perazzo y Pantoja (2020). 


\section{1. Área Académica: Economía}

Se presenta a los hombres y mujeres encuestados, siendo un total de 14.

Gráfica 3. Sexo.

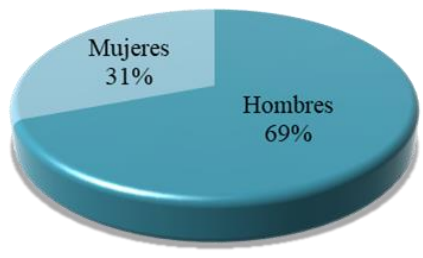

Fuente: Perazzo y Pantoja (2020).

Con respecto a la edad en la tabla 5, el $35.7 \%$ tiene entre 22 y 29 años, el $64.3 \%$ tiene entre 30-40 años y ninguno tiene más de 40 años. Al igual que en el caso de Derecho, son profesores jóvenes.

Tabla 5. Edad.

\begin{tabular}{lll}
\hline Rango/años & Frecuencia & Porcentaje \\
\hline $22-26$ & 3 & 21,4 \\
$27-29$ & 2 & 14,3 \\
$30-34$ & 3 & 21,4 \\
$35-40$ & 6 & 42,9 \\
$>40$ & 0 & 0,0 \\
Total & 14 & 100,0 \\
\hline
\end{tabular}

Fuente: Perazzo y Pantoja (2020).
En cuanto al nivel de estudios alcanzados podemos observar en la gráfica 4 que el $57 \%$ declaró tener estudios de licenciatura (tercer nivel), mientras que el $36 \%$ tiene estudios de maestría y sólo el $7 \%$ cuenta con doctorado.

Gráfica 4. Nivel de Estudios Alcanzado.

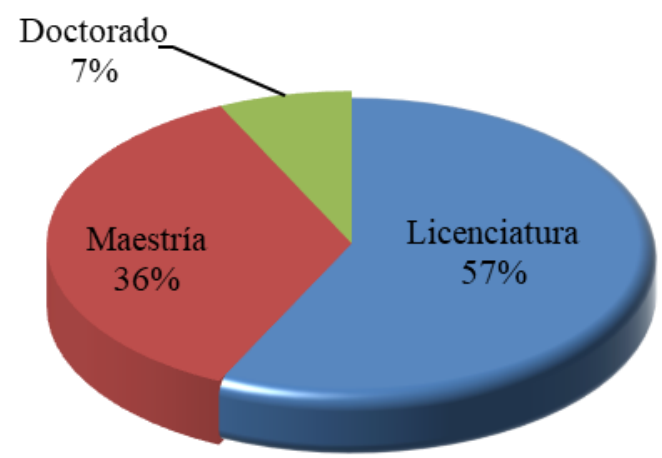

Fuente: Perazzo y Pantoja (2020).

El $71.4 \%$ de los profesores tienen una antigüedad de 1 a 5 años, mientras que el $14.3 \%$ cuenta con un tiempo de servicios de entre 6 y 12 años y sólo el $14.3 \%$ cuenta con una antigüedad de más de 12 años. Así también del total de los encuestados el $50.0 \%$ son profesores de medio tiempo, mientras que el $35.7 \%$ es de tiempo completo.

Con respecto a las tecnologías que se usan en clase, como podemos observar en la tabla 6 , sólo las transparencias tienen una moda de 4, lo cual implica que muchas veces las emplean, mientras todas las demás herramientas muestran una moda de 1 , lo cual indica que nunca las aplican. Excepto el software general para el que indicaron que algunas veces lo utilizan y la televisión para el que indicaron que pocas veces la usan. 
Tabla 6. Uso de las tecnologías en clases.

\begin{tabular}{|c|c|c|c|c|c|c|c|c|c|c|c|c|c|c|}
\hline \multirow[t]{2}{*}{ Categoría } & \multicolumn{7}{|c|}{ Porcentaje } & \multicolumn{5}{|c|}{ Frecuencia } & \multirow[t]{2}{*}{ Moda } & \multirow[t]{2}{*}{ Media } \\
\hline & 0 & 1 & 2 & 3 & 4 & 5 & 0 & 1 & 2 & 3 & 4 & 5 & & \\
\hline \multirow[t]{2}{*}{ Transparencias } & & 0,0 & 21,4 & 28,8 & 42,9 & 7,1 & 0 & 0 & 3 & 4 & 6 & 1 & 4 & 3,36 \\
\hline & 0,0 & & & & & & & & & & & & & \\
\hline Diapositivas & 0,0 & 42,9 & 21,4 & 14,3 & 14,3 & 7,1 & 0 & 6 & 3 & 2 & 2 & 1 & 1 & 2,21 \\
\hline Videogramas & 0,0 & 50,0 & 14,3 & 28,6 & 7,1 & 0,0 & 0 & 7 & 2 & 4 & 1 & 0 & 1 & 1,93 \\
\hline Filmes/Video & 0,0 & 50,0 & 0,0 & 21,4 & 14,3 & 14,3 & 0 & 7 & 0 & 3 & 2 & 2 & 1 & 2,43 \\
\hline Televisión & 0,0 & 28,6 & 14,3 & 7,1 & 28,6 & 21,4 & 0 & 4 & 2 & 1 & 4 & 3 & 2 & 3,00 \\
\hline Videodiscos & 0,0 & 64,3 & 0,0 & 21,4 & 14,3 & 0,0 & 0 & 9 & 0 & 3 & 2 & 0 & 1 & 1,86 \\
\hline Grabaciones sonoras & 0,0 & 78,6 & 14,3 & 7,1 & 0,0 & 0,0 & 0 & 11 & 2 & 1 & 0 & 0 & 1 & 1,29 \\
\hline Computadoras & 0,0 & 57,1 & 7,1 & 14,3 & 21,4 & 0,0 & 0 & 8 & 1 & 2 & 3 & 0 & 1 & 2,00 \\
\hline Materiales & 0,0 & 50,0 & 7,1 & 21,4 & 21,4 & 0,0 & 0 & 7 & 1 & 3 & 3 & 0 & 1 & 2,14 \\
\hline \multicolumn{15}{|c|}{ Hipermedia/Multimedia } \\
\hline Radio & 0,0 & 78,6 & 0,0 & 14,3 & 7,1 & 0,0 & 0 & 11 & 0 & 2 & 1 & 0 & 1 & 1,50 \\
\hline Datashow & 0,0 & 78,6 & 7,1 & 14,3 & 0,0 & 0,0 & 0 & 11 & 1 & 2 & 0 & 0 & 1 & 1,36 \\
\hline Software tutorial & 0,0 & 64,3 & 14,3 & 14,3 & 0,0 & 7,1 & 0 & 9 & 2 & 2 & 0 & 1 & 1 & 1,71 \\
\hline Power Point & 7,1 & 35,7 & 21,4 & 21,4 & 7,1 & 7,1 & 1 & 5 & 3 & 3 & 1 & 1 & 1 & 2,07 \\
\hline Software general & 7,1 & 35,7 & 0,0 & 42,9 & 14,3 & 0,0 & 1 & 5 & 0 & 6 & 2 & 0 & 3 & 2,21 \\
\hline Photoshop & 7,1 & 57,1 & 14,3 & 21,4 & 0,0 & 0,0 & 1 & 8 & 2 & 3 & 0 & 0 & 1 & 1,50 \\
\hline
\end{tabular}

Fuente: Perazzo y Pantoja (2020).

La mayoría de los objetivos como puede observarse en la tabla 7, recibieron puntuaciones altas con una moda de entre 4 y 5 , lo cual indica que muchas veces y siempre se usan las TIC para alcanzar estos objetivos. Mientras que los objetivos memorizar, relacionar/vincular/ordenar, sintetizar, propiciar nuevas relaciones, hacer conocer diversas culturas existentes y gestión de recursos, sólo se persiguen algunas veces. 
Tabla 7. Objetivos en el Uso de las TIC.

\begin{tabular}{|c|c|c|c|c|c|c|c|c|c|c|c|c|c|c|}
\hline \multirow[t]{2}{*}{ Categoría } & \multicolumn{6}{|c|}{ Porcentaje } & \multicolumn{6}{|c|}{ Frecuencia } & \multirow[t]{2}{*}{ Moda } & \multirow[t]{2}{*}{ Media } \\
\hline & 0 & 1 & 2 & 3 & 4 & 5 & 0 & 1 & 2 & 3 & 4 & 5 & & \\
\hline Motivar e interesar & 7,1 & 0,0 & 14,3 & 21,4 & 28,6 & 28,6 & 1 & 0 & 2 & 3 & 4 & 4 & 5 & 3,50 \\
\hline Percibir lo complejo y lo abstracto & 7,1 & 0,0 & 14,3 & 0,0 & 57,1 & 21,4 & 1 & 0 & 2 & 0 & 8 & 3 & 4 & 3,64 \\
\hline Documentar & 7,1 & 0,0 & 14,3 & 14,3 & 35,7 & 28,6 & 1 & 0 & 2 & 2 & 5 & 4 & 4 & 3,57 \\
\hline Memorizar & 7,1 & 21,4 & 14,3 & 35,7 & 7,1 & 14,3 & 1 & 3 & 2 & 5 & 1 & 2 & 3 & 2,57 \\
\hline Nuevos aspectos & 7,1 & 0,0 & 14,3 & 21,4 & 35,7 & 21,4 & 1 & 0 & 2 & 3 & 5 & 3 & 4 & 3,43 \\
\hline Relacionar/Vincular/Ordenar & 7,1 & 0,0 & 0,0 & 42,9 & 21,4 & 28,6 & 1 & 0 & 0 & 6 & 3 & 4 & 3 & 3,57 \\
\hline Clases atractivas & 7,1 & 0,0 & 0,0 & 14,3 & 35,7 & 42,9 & 1 & 0 & 0 & 2 & 5 & 6 & 5 & 4,00 \\
\hline Transmitir perspectivas distintas & 7,1 & 0,0 & 7,1 & 21,4 & 35,7 & 28,6 & 1 & 0 & 1 & 3 & 5 & 4 & 4 & 3,64 \\
\hline Concientizar & 7,1 & 0,0 & 7,1 & 7,1 & 64,3 & 14,3 & 1 & 0 & 1 & 1 & 9 & 2 & 4 & 3,64 \\
\hline Investigar & 7,1 & 0,0 & 0,0 & 14,3 & 42,9 & 35,7 & 1 & 0 & 0 & 2 & 6 & 5 & 4 & 3,93 \\
\hline Sintetizar & 7,1 & 7,1 & 7,1 & 42,9 & 35,7 & 0,0 & 1 & 1 & 1 & 6 & 5 & 0 & 3 & 2,93 \\
\hline Retroalimentar & 7,1 & 0,0 & 0,0 & 21,4 & 57,1 & 14,3 & 1 & 0 & 0 & 3 & 8 & 2 & 4 & 3,64 \\
\hline Generar creatividad & 7,1 & 0,0 & 0,0 & 28,6 & 35,7 & 28,6 & 1 & 0 & 0 & 4 & 5 & 4 & 4 & 3,71 \\
\hline Trabajo colaborativo & 7,1 & 0,0 & 0,0 & 21,4 & 28,6 & 42,9 & 1 & 0 & 0 & 3 & 4 & 6 & 5 & 3,93 \\
\hline Propiciar nuevas relaciones & 7,1 & 0,0 & 0,0 & 50,0 & 14,3 & 28,6 & 1 & 0 & 0 & 7 & 2 & 4 & 3 & 3,59 \\
\hline Formación y perfeccionamiento & 7,1 & 0,0 & 7,1 & 14,3 & 50,0 & 21,4 & 1 & 0 & 1 & 2 & 7 & 3 & 4 & 3,64 \\
\hline $\begin{array}{l}\text { Hacer conocer diversas culturas } \\
\text { existentes }\end{array}$ & 7,1 & 0,0 & 7,1 & 42,9 & 28,6 & 14,3 & 1 & 0 & 1 & 6 & 4 & 2 & 3 & 3,29 \\
\hline Propiciar uso de internet & 7,1 & 0,0 & 14,3 & 14,3 & 35,7 & 28,6 & 1 & 0 & 2 & 2 & 5 & 4 & 4 & 3,57 \\
\hline Gestión de recursos & 0,0 & 7,1 & 7,1 & 64,3 & 21,4 & 0,0 & 0 & 1 & 1 & 9 & 3 & 0 & 3 & 3,00 \\
\hline Organización de clases & 0,0 & 7,1 & 7,1 & 14,3 & 50,0 & 21,4 & 0 & 1 & 1 & 2 & 7 & 3 & 4 & 3,71 \\
\hline Evaluar & 7,1 & 7,1 & 7,1 & 28,6 & 35,7 & 14,3 & 1 & 1 & 1 & 4 & 5 & 2 & 4 & 3,21 \\
\hline
\end{tabular}

Fuente: Perazzo y Pantoja (2020).

La valoración de las actitudes pedagógicas en relación con las TIC muestra que los profesores están muy de acuerdo con la mayoría de las actitudes señaladas en la tabla 8. Mientras que se mostraron siempre de acuerdo con que el profesor prepara los recursos didácticos necesarios y algunas veces de acuerdo con que el aprendizaje se evalúa a partir de la aplicación de conocimientos. 
Tabla 8. Actitudes de los profesores en relación con las TIC.

\section{Categoría}

Porcentaje

Frecuencia

Moda Media

\begin{tabular}{|c|c|c|c|c|c|c|c|c|c|c|c|c|c|c|}
\hline & 0 & 1 & 2 & 3 & 4 & 5 & 0 & 1 & 2 & 3 & 4 & 5 & & \\
\hline $\begin{array}{l}\text { La enseñanza es causa } \\
\text { directa del aprendizaje }\end{array}$ & 0,0 & 0,0 & 0,0 & 35,7 & 50,0 & 14,3 & 0 & 0 & 0 & 5 & 7 & 2 & 4 & 3,79 \\
\hline $\begin{array}{l}\text { El aprendizaje se mide con } \\
\text { relación a reglas/patrones }\end{array}$ & 0,0 & 0,0 & 14,3 & 28,6 & 35,7 & 21,4 & 0 & 0 & 2 & 4 & 5 & 3 & 4 & 3,64 \\
\hline $\begin{array}{l}\text { El aprendizaje es resultado } \\
\text { de un conjunto de procesos }\end{array}$ & 0,0 & 0,0 & 0,0 & 14,3 & 57,1 & 28,8 & 0 & 0 & 0 & 2 & 8 & 4 & 4 & 4,14 \\
\hline $\begin{array}{l}\text { El aprendizaje se evalúa a } \\
\text { partir de la aplicación de } \\
\text { conocimientos }\end{array}$ & 0,0 & 0,0 & 7,1 & 35,7 & 28,6 & 28,6 & 0 & 0 & 1 & 5 & 4 & 4 & 3 & 3,79 \\
\hline $\begin{array}{l}\text { Enseñar bien es lo bastante } \\
\text { para aprender bien }\end{array}$ & 0,0 & 7,1 & 0,0 & 14,3 & 42,9 & 35,7 & 0 & 1 & 0 & 3 & 6 & 5 & 4 & 4,00 \\
\hline $\begin{array}{l}\text { Usar la técnica más } \\
\text { apropiada no es sinónimo } \\
\text { de eficacia }\end{array}$ & 0,0 & 0,0 & 21,4 & 28,6 & 28,6 & 21,4 & 0 & 0 & 3 & 4 & 4 & 3 & 4 & 3,50 \\
\hline $\begin{array}{l}\text { El profesor prepara } \\
\text { pormenorizadamente las } \\
\text { respuestas }\end{array}$ & 0,0 & 7,1 & 0,0 & 21,4 & 50,0 & 21,4 & 0 & 1 & 0 & 3 & 7 & 3 & 4 & 3,79 \\
\hline $\begin{array}{l}\text { Contenidos más cercanos } \\
\text { aprendizaje más eficaz }\end{array}$ & 0,0 & 7,1 & 7,1 & 7,1 & 42,9 & 35,7 & 0 & 1 & 1 & 1 & 6 & 5 & 4 & 3,93 \\
\hline $\begin{array}{l}\text { Técnicas bien aplicadas } \\
\text { garantizan la eficacia }\end{array}$ & 0,0 & 7,1 & 7,1 & 7,1 & 50,0 & 28,6 & 0 & 1 & 1 & 1 & 7 & 4 & 4 & 3,86 \\
\hline $\begin{array}{l}\text { El profesor prepara los } \\
\text { recursos } \\
\text { necesarios }\end{array}$ & 0,0 & 7,1 & 0,0 & 7,1 & 35,7 & 50,0 & 0 & 1 & 0 & 1 & 5 & 7 & 5 & 4,21 \\
\hline
\end{tabular}

Fuente: Perazzo y Pantoja (2020). 


\section{DISCUSIÓN}

\section{Necesidad de la formación del profesorado en las TIC}

Para Pantoja (1995):

La formación docente, es un proceso sistemático, abierto y permanente cuyo propósito, es el de formar a los profesores para que adquieran las competencias docentes y profesionales necesarias para el desarrollo de su práctica docente y profesional bajo los lineamientos teóricos y metodológicos de las actuales corrientes didácticas, sociales, psicológicas, que le posibiliten, el transformar su entorno social, político y económico.

A la luz de los resultados obtenidos, en este caso la formación del profesorado en TIC implica actividades que incidan en el "perfeccionamiento de la función docente" y por lo tanto, en el aumento de la calidad de la enseñanza. Deberá gestionarse al interior de la institución una formación en TIC que permita desarrollar la competencia digital de manera continua, lo cual implica como lo refiere Marcelo (1989) “...como un proceso de desarrollo profesional en el que la formación inicial es la primera fase (interconexión entre la formación inicial y el currículum de la formación permanente) y la integración de contenidos propiamente académicos-disciplinares y la formación pedagógica de los profesores".

La formación del profesorado adquiere un interés cada vez mayor y supone un desafío para el formador, hay interés por parte de la tecnología educativa en promover:

a) La formación de docentes para el uso de las TIC.

b) La formación de docentes con TIC.

c) El análisis de las actitudes de los profesores ante la tecnología educativa.

d) Los enfoques metodológicos para la preparación inicial y permanente de los profesores en las modalidades de la utilización de la tecnología educativa.

La metodología aplicada en la formación de los profesores debe asumir el repertorio de técnicas que se desea que el alumno aplique en su futuro ejercicio profesional. Con lo cual la formación de profesores en TIC hace posible:

1. Una mejor estructura del contenido.

2. Estandarizar ciertas decisiones en materia educativa.

3. Hacer más formal la instrucción.

4. Rentabilizar el tiempo dedicado a la enseñanza.

5. Promover una enseñanza más interactiva.

6. Flexibilizar el tiempo dedicado a la enseñanza.

7. Cambiar el rol del profesor, de enseñante a facilitador.

Con la necesidad de implementar la tecnología en el aula, urge entonces la obligación de capacitar a los profesores en el dominio de las TIC, a través de una pedagogía de la imagen y del uso racional y crítico de los recursos tecnológicos en su aplicación a la educación. He ahí los retos a los que se tienen que afrontar las instituciones educativas en la formación de los docentes, por lo tanto, será preciso contar con un elevado número de profesores en los ámbitos de formación.

Hemos de entender y así lo confirman algunos estudios realizados por Grau (1996) que la decisión de cada profesor, por utilizar innovaciones tecnológicas, está asociada a sus propias perspectivas de valores (esfuerzo, tiempo, riesgo) y compensaciones de tipo profesional, ya sea simbólicas, no económicas o pedagógicas, mejorar su imagen como docente, mejorar la relación con los alumnos, beneficio del aprendizaje de los alumnos, entre otras.

En un intento por resolver este dilema, podemos plantear la siguiente ecuación:

V.I. $=C-(E+R)$

Dónde:

V.I. = Valor de la innovación tecnológica a tener en cuenta en la decisión que adopte el profesor. 
$\mathrm{C}=$ Compensaciones o recompensas que pueda obtener (simbólicas o pedagógicas).

$\mathrm{E}=$ Los esfuerzos que conllevan al hecho de innovar tecnológicamente, o sea, el tiempo y la capacitación profesional.

$\mathrm{R}=$ Los riesgos que entraña la inclusión de la innovación tecnológica en el aula.

Es decir que el valor de cualquier innovación vendrá determinado por la diferencia entre las compensaciones y recompensas que el profesor pueda obtener y los esfuerzos y riesgos que tal innovación entrañe.

\section{Como afirma López (2017, p. 301)}

Sin duda el uso que las TIC ha tenido en la educación está estrechamente relacionado con la mente de los usuarios. En principio a estas herramientas se les vio como máquinas para procesar datos; posteriormente se les asoció con el uso de programas de propósito general o de productividad. Más adelante permitió el desarrollo de los programas multimedia. En las décadas de 1980 y 1990 a las TIC se les pudo ver como auténticas herramientas cognitivas. En la actualidad y con el uso de internet, las TIC ayudan a conformar complejas comunidades virtuales en las que sobresale el desarrollo de una inteligencia colectiva, sumado el talento y el potencial de muchos, en una sola aplicación.

\section{CONCLUSIONES}

La necesidad de formación del profesorado a través de las TIC para su aplicación en el aula se avanzará a medida que se haga extensiva su labor en la institución educativa, mediante la formación a través de formadores que reproduzcan y adapten las experiencias de formación en este aspecto a su trabajo en el aula.

Es importante se forme un educador idóneo con vocación por el hombre, que esté capacitado para trabajar en diferentes realidades de vida, en otras palabras, un profesional de la educación efectivo y pertinente que se caracterice por:

* Ser científico, en el sentido de ser capaz de diagnosticar, evaluar situaciones y proponer acciones que le permitan ajustarse a la realidad en que le corresponde trabajar.
*Tener una formación axiológica tal, que le permita actuar conforme a valores universales, como el respeto, la libertad, la solidaridad, la participación, y la tolerancia, entre otros.

* Desarrollar en las personas los procesos de pensamiento que le permitan "aprender a aprender" y actuar creativamente.

* Proceder interdisciplinariamente para enfrentar los problemas educativos de la comunidad en que trabaja y que exceden su formación profesional.

*Tener un alto grado de autoestima y sentirse seguro de su rol profesional.

* Ser una persona con una actitud fundamentalmente humanista, que se dé a conocer, que valore a los otros y por ende a su propia realidad.

*Ser un hombre que se brinde "con el otro y para el otro", que facilite el desarrollo de cada alumno, como ser único e irrepetible, hasta su máxima expresión, sin descuidar la dimensión espiritual y afectiva.

* Poseer capacidad para comunicarse, comprender, respetar y valorar la diversidad cultural de sus alumnos.

Las estrategias que posibilitan el logro de las características anteriores son:

- Que experimente un real proceso de personalización, a través de experiencias vivenciales en las áreas afectiva, cognoscitiva y psicomotora, en un clima que favorezca la libre integración del educador y del educando.

- Lograr una visión profunda e integrada del mundo circundante.

- Enfrentar opiniones que impliquen cambios y acepten los desafíos ante las TIC.

Disponer de tecnologías eficientes que propicien la búsqueda del conocimiento sistemático en forma autónoma y creativa. 


\section{REFERENCIAS BIBLIOGRÁFICAS}

Grau, J. (1996). La Informática Educativa en el Marco de la Educación. Escuela Universitaria de Magisterio de Toledo; XXI.

Hernández, L.; Acevedo, J.; Martínez, C. \& Cruz, B. (2014). El uso de las TIC en el aula: un análisis en términos de efectividad y eficacia. Congreso Iberoamericano de Ciencia, Tecnología, Innovación y Educación; 121.

Google Scholar

López, M. (2017) Aprendizaje, competencias y TIC. Pearson: México.

Marcelo, C. (1989). Introducción a la formación del profesorado. Universidad de Sevilla: Sevilla, España.

Google Scholar

Ortiz, F. (2016) Diccionario de Metodología de la Investigación Científica. Limusa: México.

Pantoja Sánchez, M. (1995). Propuesta de un Diplomado en Docencia Universitaria a Distancia para Profesores de las Facultades de Contaduría y Administración en las Universidades del Interior de la República Mexicana. Tesis de Maestría en Tecnología de la Educación. Universidad de Salamanca: Salamanca, España.

Prats, J. (2016). Internet en las aulas. Mamakuna; 2; 60-66. Universidad Nacional de Educación: Ecuador.

\section{Google Scholar}

Perazzo, D. (1995). Propuesta de un Modelo Explicativo para la Innovación en los Centros Educativos. I Congreso de Innovación Educativa; I; 365-370. Universidad de Santiago de Compostela: Galicia, España.
Salvador, A. (1991). La Informática en la Acción Educativa. M.E.C. Madrid, España.
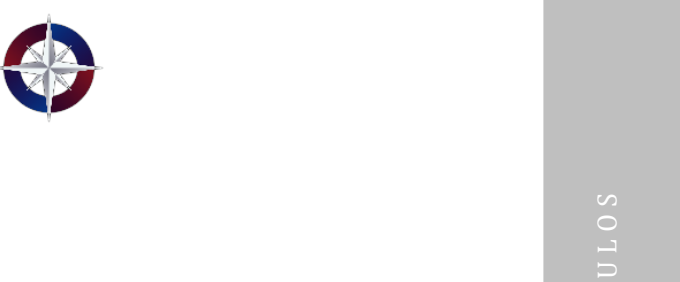